\title{
Colonoscopy quality across Europe: a report of the European Colonoscopy Quality Investigation (ECQI) Group
}

\section{(ㄷ)(1) $\odot$}

\section{Authors}

Cristiano Spada ${ }^{1,2}$, Anastasios Koulaouzidis ${ }^{3}{ }^{\circ}$, Cesare Hassan ${ }^{4} \odot$, Pedro Amaro ${ }^{5}$, Anurag Agrawal ${ }^{6}$, Lene Brink ${ }^{7}$, Wolfgang Fischbach ${ }^{8}$, Matthias Hünger ${ }^{9}$, Rodrigo Jover ${ }^{10}$, Urpo Kinnunen ${ }^{11}$, Akiko Ono ${ }^{12}$, Árpad Patai ${ }^{13}$, Silvia Pecere $^{14} \odot$, Lucio Petruzziello ${ }^{14}$, Jürgen F. Riemann ${ }^{15}$, Bharat Amlani ${ }^{16}$, Harry Staines ${ }^{17}$, Ann L. Stringer ${ }^{18}$, Ervin Toth $^{19} \odot$, Giulio Antonelli ${ }^{4,20,21}$, Lorenzo Fuccio ${ }^{22}$

Institutions

1 Digestive Endoscopy Unit and Gastroenterology, Fondazione Poliambulanza, Brescia, Italy

2 Digestive Endoscopy Unit, Università Cattolica del Sacro Cuore, Rome, Italy

3 Pomeranian Medical University in SzczecinDepartment of Social Medicine and Public Health, Faculty of Health Sciences, Szczecin, Zachodniopomorskie, Poland

4 Digestive Endoscopy, Nuovo Regina Margherita Hospital, Rome, Italy

5 Gastroenterology Department, Centro Hospitalar e Universitário de Coimbra, Coimbra, Portugal

6 Doncaster Royal Infirmary, Doncaster, UK

7 Herlev and Gentofte Hospital, Copenhagen University, Gastro Unit, Division of Endoscopy, Herlev, Denmark

8 Gastroenterologie und Innere Medizin, Aschaffenburg, Germany

9 Private Practice for Internal Medicine, Würzburg, Germany

10 Hospital General Universitario de Alicante - Instituto de Investigación Sanitaria ISABIAL - Servicio de Medicina Digestiva, Alicante, Spain

11 Tampere University Hospital-Gastroenterology, Tampere, Finland

12 Hospital Clínico Universitario Virgen de la ArrixacaGastroenterology, El Palmar, Murcia, Spain

13 Markusovszky University Teaching HospitalGastroenterology, Szombathely, Hungary

14 Digestive Endoscopy Unit, Fondazione Policlinico Universitario A. Gemelli IRCCS, Rome, Italy

15 LebensBlicke Foundation, Ludwigshafen, Germany

16 Norgine Ltd-Medical Affairs, Harefield, UK

17 Sigma Statistical Services Ltd, Saint Andrews, UK

18 ECQI Secretariat, Buckinghamshire, UK

19 Skåne University Hospital, Lund University, Department of Gastroenterology, Malmö, Sweden

20 Department of Anatomical, Histological, Forensic Medicine and Orthopedics Sciences, "Sapienza” University of Rome, Rome, Italy
21 Gastroenterology and Digestive Endoscopy Unit, Ospedale dei Castelli Hospital, Ariccia, Rome, Italy

22 Gastroenterology Unit, Department of Medical and Surgical Sciences, S. Orsola-Malpighi Hospital, Bologna, Italy

submitted 15.10.2020

accepted after revision 10.3.2021

Bibliography

Endosc Int Open 2021; 09: E1456-E1462

DOI 10.1055/a-1486-6729

ISSN 2364-3722

(c) 2021. The Author(s).

This is an open access article published by Thieme under the terms of the Creative Commons Attribution-NonDerivative-NonCommercial License, permitting copying and reproduction so long as the original work is given appropriate credit. Contents may not be used for commercial purposes, or adapted, remixed, transformed or built upon. (https://creativecommons.org/licenses/by-nc-nd/4.0/)

Georg Thieme Verlag KG, Rüdigerstraße 14,

70469 Stuttgart, Germany

Corresponding author

Cristiano Spada, Fondazione Poliambulanza - Digestive

Endoscopy Unit and Gastroenterology, Via L. Bissolati 57,

25124 Brescia, Italy

Fax: +390303518221

cristianospada@gmail.com

Supplementary material is available under https://doi.org/10.1055/a-1486-6729

\section{ABSTRACT}

Background and study aims The European Colonoscopy Quality Investigation (ECQI) Group comprises expert colonoscopists and investigators with the aim of raising colonoscopy standards. We assessed the levels of monitoring and achievement of European Society of Gastrointestinal Endoscopy (ESGE) performance measures (PMs) across Europe using responses to the ECQI questionnaires. 
Methods The questionnaire comprises three forms: institution and practitioner questionnaires are completed once; a procedure questionnaire is completed on multiple occasions for individual total colonoscopies. ESGE PMs were approximated as closely as possible from the data collected via the procedure questionnaire. Procedure data could provide rate of adequate bowel preparation, cecal intubation rate (CIR), withdrawal time, polyp detection rate (PDR), and tattooing resection sites.

Results We evaluated ECQI questionnaire data collected between June 2016 and April 2018, comprising 91 practitioner and 52 institution questionnaires. A total of 6445 completed procedure forms were received.
Institution and practitioner responses indicate that routine recording of PMs is not widespread: adenoma detection rate (ADR) is routinely recorded in $29 \%$ of institutions and by $34 \%$ of practitioners; PDR by $42 \%$ and $47 \%$, CIR by $62 \%$ and $64 \%$, bowel preparation quality by $56 \%$ and $76 \%$, respectively.

Procedure data showed a rate of adequate bowel preparation of $84.2 \%$, CIR $73.4 \%$, PDR $40.5 \%$, mean withdrawal time 7.8 minutes and $12.2 \%$ of procedures with possible removal of a non-pedunculated lesion $\geq 20 \mathrm{~mm}$ reporting tattooing.

Conclusions Our findings clearly show areas in need of quality improvement and the importance of promoting quality monitoring throughout the colonoscopy procedure.

\section{Introduction}

Colonoscopy has been shown to greatly reduce colorectal cancer (CRC) incidence and mortality as it allows for both identification of early neoplasia and removal of precancerous lesions $[1,2]$. While adenoma detection rate (ADR) is considered a primary quality indicator $[3,4]$, it is dependent on other quality measures, such as cecal intubation rate (CIR), withdrawal time, and quality of bowel preparation [5].

There is considerable variability in the quality of colonoscopy [6] with a three- to six-fold variation in ADR among endoscopists [7,8]. Given the substantial impact of CRC on patients and healthcare systems $[9,10]$, and that screening can be effective provided the services are of high quality [11], it is clearly important to ensure that colonoscopy is delivered to a high standard across the endoscopy community.

The European Society of Gastrointestinal Endoscopy (ESGE) has published both performance measures (PMs) for lower gastrointestinal endoscopy [12] and PMs for the endoscopy service as a whole [13], providing to all stakeholders (patients and their advocacy groups; service leaders; staff, including endoscopists; professional societies; payers and regulators) recommendations on the necessary parameters needed to meet the requirements of the ESGE quality improvement initiatives. These measures include those related to leadership, organisation, and service delivery, as well as those associated with the patient journey, and comprise recommendations for a minimum and target standard for endoscopy services to achieve. A crucial aspect of the guidelines is periodical monitoring of PMs both on individual practitioners, as well as at institutional service levels.

The European Colonoscopy Quality Investigation (ECQI) Group comprises expert colonoscopists and investigators with the aim of raising colonoscopy standards across Europe. ECQI does not wish to create any specific quality criteria, but rather document how the recent ESGE guidelines are implemented in daily practice and assess the quality of colonoscopy practice in Europe. We aimed to assess the levels of monitoring and achievement of ESGE PMs across Europe using responses to the ECQI questionnaires.

\section{Methods}

At the inaugural meeting of the ECQI Group in 2013, the Group chose to develop a clinical practice questionnaire to enable colonoscopists to evaluate current practice. The online questionnaire was based on the ESGE position statement on quality in screening colonoscopy published in 2012 [14]. An iterative process was used to hone the questionnaire ensuring that the time to complete the form was not too onerous. It was validated in November 2014 and May 2015 during two pilot phases, via a collaborative approach to ensure pertinent information was being recorded and data on 1861 patient procedures were collected $[15,16]$. The questionnaire comprises three forms: institution (18 questions) and practitioner questionnaires (12 questions) are each completed once, recording routine practice at respective levels; a procedure questionnaire (34 questions) is completed on multiple occasions for individual total colonoscopies (see Supplementary Material).

Participation was open to all Europe-based colonoscopists via web-based registration at the ECQI Group website. Awareness of the questionnaire came from abstracts, posters, presentations at national and international congresses and individual communications from ECQI Group members. Interested participants applied via the ECQI Group website or to the ECQI Group Secretariat. Following verification, log-in access to the web-based questionnaire site was provided by email.

\section{Calculation of performance measures}

ESGE PMs [12] were approximated as closely as possible from the data collected via the procedure questionnaire. We determined that our questionnaires could provide approximations for rate of adequate bowel preparation, CIR, withdrawal time, polyp detection rate (PDR), and tattooing resection sites ( $\bullet$ Table 1). For tattooing, we were unable to include polyps with suspicious macroscopic features regardless of size, as we were limited in the ability to determine the presence of suspicious macroscopic features due to questionnaire design, so this measure only includes procedures with a non-pedunculated polyp $\geq 20 \mathrm{~mm}$. We were also unable to determine from ques- 
- Table1 Definitions used for calculation of performance measures [12].

\begin{tabular}{|c|c|c|}
\hline Performance measure & Numerator & Denominator \\
\hline $\begin{array}{l}\text { Rate of adequate bowel } \\
\text { preparation }\end{array}$ & Patients with $B B P S \geq 6$ & Patients undergoing colonoscopy \\
\hline Cecal intubation rate & $\begin{array}{l}\text { Procedures that report reaching the cecum (docu- } \\
\text { mented in written form and by photo/video) }\end{array}$ & $\begin{array}{l}\text { All screening or diagnostic colonoscopies with indication to } \\
\text { reach the cecum }\end{array}$ \\
\hline Withdrawal time & Sum of withdrawal times included in denominator & $\begin{array}{l}\text { Number of negative (no biopsy/therapy) screening or diag- } \\
\text { nostic colonoscopies, excluding incomplete colonoscopies }\end{array}$ \\
\hline Polyp detection rate & Procedures with at least one polyp identified & $\begin{array}{l}\text { All screening and diagnostic colonoscopies in patients aged } \\
50 \text { years or older }\end{array}$ \\
\hline Tattooing resection sites & $\begin{array}{l}\text { Procedures where the resection site was marked } \\
\text { with a tattoo }\end{array}$ & $\begin{array}{l}\text { Colonoscopies with removal of non-pedunculated lesions } \\
20 \mathrm{~mm} \text { in size or larger }\end{array}$ \\
\hline Polyp removal rate $(>5 \mathrm{~mm})^{1}$ & Procedures with an endoscopic intervention & All procedures with a polyp $>5 \mathrm{~mm}$ reported \\
\hline
\end{tabular}

tionnaire responses whether tattooing was performed on the resection site; we were able to determine whether it was performed during the same procedure as an endoscopic intervention that could have resulted in polyp removal. We also provide an indication of polyp removal rate for procedures with a polyp $>5 \mathrm{~mm}$, as our questionnaire was unable to determine the polyp retrieval rate for histopathology examination.

A score of $\geq 6$ on the Boston Bowel Preparation Scale (BBPS) was used to define adequate bowel preparation [17]. In procedures with data missing for one segment, if it could not be determined that the BBPS was definitely either $\geq 6$ or $<6$, procedures were classified as missing, along with all other procedures with more than one segment missing data. To calculate CIR, only those procedures reporting the cecum as the intended endpoint were included, which excluded some procedures with terminal ileum/neo-terminal ileum as the intended endpoint, because given the questionnaire design, we could not determine whether the cecum was photo-documented in these procedures.

Diagnostic and screening procedures were determined using the reason that was provided for performing them. Our questionnaire had no method of collecting histopathological data so ADR could not be calculated. Polyp detection was regarded as positive if either a polyp or a polypectomy was reported. Age at the date of the procedure was derived assuming the date of birth was June 30 (to preserve anonymity, only the patient's year of birth was recorded).

Calculation of mean withdrawal time was restricted to those procedures (screening or diagnostic) in which the cecum was the intended endpoint, the endpoint was reached, and no endoscopic intervention was reported. Procedures with a definite non-pedunculated lesion $\geq 20 \mathrm{~mm}$ were identified when in any segment reporting a polyp $\geq 20 \mathrm{~mm}$, only non-pedunculated classifications were recorded for that segment. This may have excluded some non-pedunculated lesions $\geq 20 \mathrm{~mm}$, as it excluded procedures in which both pedunculated and nonpedunculated lesions were reported in a segment with a polyp $\geq 20 \mathrm{~mm}$. We only included procedures in which an endoscopic intervention that could have removed the polyp was reported, i. e. endoscopic mucosal resection, endoscopic submucosal dissection, polypectomy (complete or incomplete) or biopsy.

\section{Results}

We evaluated ECQI questionnaire data collected between June 2, 2016 and April 30, 2018, comprising 91 completed practitioner questionnaires and 52 completed institution questionnaires from 12 European countries. A total of 6445 completed procedure forms were received from 25 academic hospitals (2270/6445, 35.2\%), 14 hospitals (1235/6445, 19.1\%), eight private institutions $(2657 / 6445,41.2 \%)$, three group practices (160/6445, $2.5 \%)$, and one other $(123 / 6445,1.9 \%)$. Results are summarized in $>$ Table 2 and $>$ Table 3.

\section{Pre-procedure}

A reason for colonoscopy was provided for 6413 of 6445 procedures (99.5\%). These were classified as: diagnostic (3182/6445, $49.3 \%)$, screening (1274/6445, 19.8\%), follow-up (1837/6445; $28.5 \%$ ), previous unsuccessful procedure (99/6445, $1.5 \%$ ), and other $(21 / 6445,0.3 \%)$. Screening was classified as "due to familial risk" in $29.7 \%$ (378/1274) of procedures, "following a positive screening test" in $39.2 \%(499 / 1274)$ and "without a prescreening test" in 30.9\% (394/1274), and one other.

The collected responses showed that scale-based bowel cleansing quality was reported as "routinely recorded" by $56 \%$ of institutions and "routinely used" by $76 \%$ of practitioners. From the procedure data, $84.2 \%$ (5427/6445) of procedures reported an adequate bowel cleansing (data missing for 209 procedures, $3.2 \%)$.

\section{Completeness of procedure}

CIR was reported as "routinely recorded" by $64 \%$ of practitioners and by $62 \%$ of institutions. Procedure data showed that the cecum was the intended endpoint in $69.4 \%(4473 / 6445)$ of 
- Table 2 Proportion of institutions and practitioners that routinely record performance measures.

\begin{tabular}{|l|l|l|}
\hline Performance measure & $\begin{array}{l}\text { Institutions } \\
\mathbf{N}=\mathbf{5 2}\end{array}$ & $\begin{array}{l}\text { Practitioners } \\
\mathbf{N}=\mathbf{9 1}\end{array}$ \\
\hline $\begin{array}{l}\text { Rate of adequate bowel prepara- } \\
\text { tion }\end{array}$ & $56 \%$ & $76 \%$ \\
\hline Cecal intubation rate & $62 \%$ & $64 \%$ \\
\hline Retraction time & NA & $60 \%$ \\
\hline Adenoma detection rate & $29 \%$ & $34 \%$ \\
\hline Polyp detection rate & $42 \%$ & $47 \%$ \\
\hline Polyp removal rate & NA & $44 \%$ \\
\hline Polyp retrieval rate & NA & $37 \%$ \\
\hline Use of a polyp classification scale & NA & $77 \%$ \\
\hline Tattooing based on guidelines & NA & $54 \%$ \\
\hline Patient satisfaction & $25 \%$ & NA \\
\hline During-procedure complications & $83 \%$ & NA \\
\hline Post-procedure complications & $56 \%$ & NA \\
\hline NA, not asked. & & \\
\hline
\end{tabular}

procedures (ileum $28.1 \%$, anastomosis $1.3 \%$, data unavailable $1.2 \%)$. For those colonoscopies in which the cecum was the intended endpoint, $94.7 \%$ reported reaching the cecum but only $77.5 \%(3281 / 4234)$ of those stated endpoint photo-documentation.

\section{Identification of pathology}

ADR was reported as "routinely recorded" by only $34 \%$ of practitioners and in $29 \%$ of institutions. PDR was "routinely recorded" by $47 \%$ of practitioners and in $42 \%$ of institutions. Retraction time was "routinely recorded" by $60 \%$ of practitioners.

At least one polyp was detected in $40.5 \%$ (1363/3365) of qualifying procedures. Withdrawal time was assessed in the 1150 qualifying procedures providing data, the overall mean $( \pm \mathrm{SD})$ withdrawal time was $7.8 \pm 3.1$ minutes, the median withdrawal time was 7 minutes.
- Table4 Endoscopic interventions in procedures with polyps $>5 \mathrm{~mm}$ (multiple options possible).

\begin{tabular}{|l|c|}
\hline Type of endoscopic intervention & $\begin{array}{l}\text { No. polyps } \\
\mathbf{N}=\mathbf{1 1 5 6}\end{array}$ \\
\hline Endoscopic mucosal resection & 154 \\
\hline Endoscopic submucosal dissection & 14 \\
\hline Polypectomy (complete) & 1012 \\
\hline Polypectomy (incomplete) & 18 \\
\hline Argon plasma coagulation & 4 \\
\hline Biopsy & 41 \\
\hline Tattooing & 40 \\
\hline Note: Biopsies performed were not necessarily related to polyp resection. \\
\hline
\end{tabular}

\section{Management of pathology}

The proportion of practitioners reporting "routinely recording" polyp removal rate was $44 \%$ and the polyp retrieval rate was $37 \%$. Routine use of a polyp classification scale was reported by $77 \%$ of practitioners and $54 \%$ routinely placed tattoos following polyp removal based on guidelines.

In the 1294 procedures where a polyp $>5 \mathrm{~mm}$ was reported, $89.3 \%$ (1156/1294) reported an endoscopic intervention ( $\triangleright$ Table 4). In procedures in which a non-pedunculated lesion $\geq 20 \mathrm{~mm}$ could be definitively identified and an endoscopic intervention to remove the polyp reported, $12.2 \%$ (17/139) reported tattooing.

\section{Complications, patient experience and post-procedure}

Patient satisfaction was recorded in $25 \%$ of institutions, duringprocedure complications were reported to be "routinely recorded" in $83 \%$, but post-procedure complications by only $56 \%$. Quality guidelines were reported to be "routinely followed" in $69 \%$ of institutions.

- Table 3 Summary of evaluated performance measures.

\begin{tabular}{|l|l|l|l|}
\hline Performance measure & ESGE minimum standard & ESGE target standard & ECQI findings \\
\hline Rate of adequate bowel preparation & $\geq 90 \%$ & $\geq 95 \%$ & $84.2 \%$ \\
\hline Cecal intubation rate & $\geq 90 \%$ & $\geq 95 \%$ & $73.4 \%(94.7 \%$ with written documentation) \\
\hline Withdrawal time & Mean 6 minutes & Mean 10 minutes & Mean 7.8 minutes \\
\hline Polyp detection rate & $40 \%$ & None set & $40.5 \%$ \\
\hline $\begin{array}{l}\text { Tattooing resection sites } \\
\text { (non-pedunculated } \geq 20 \mathrm{~mm})\end{array}$ & Unknown & $100 \%$ & $12.2 \%$ \\
\hline Polyp removal rate $(>5 \mathrm{~mm})$ & - & - & $89.3 \%$ \\
\hline ESGE, European Society of Gastrointestinal Endoscopy. & & \\
\hline
\end{tabular}




\section{Discussion}

In this study, we sought to evaluate the adoption of colonoscopy PMs across Europe. In 2017, the ESGE published PMs for lower gastrointestinal endoscopy [12], recommending that endoscopy services across Europe should adopt a list of key and minor PMs for objective assessment and evaluation in daily practice at both center and individual endoscopist level. Several key performance indicators have been established for adoption to achieve consistently high-quality endoscopic practice. We analysed a sample of procedures conducted across Europe, between June 2016 and April 2018, spanning a period before and after publication of the PMs, to evaluate the baseline achievement of standards, as defined by the ESGE. The analysis was performed at institution, practitioner and procedure levels. A set of variables listed in the ESGE lower gastrointestinal endoscopy PM document was considered. Interestingly, although some of the PMs seem to be relatively commonly assessed, documentation of other relevant PMs is far from routine.

Scale-based bowel cleansing quality was reported as routinely recorded in only $56 \%$ of institutions, and an adequate level was achieved in $84.2 \%$ of procedures, slightly below the $\geq 90 \%$ minimum standard as recommended by ESGE [12]. The quality of bowel preparation is crucial for the overall efficacy of colonoscopy, with a suboptimal ADR and CIR related to an inadequate cleansing level and a higher risk of interval cancer. [18, 19] In addition, a suboptimal cleansing level results in further costs and organizational issues since colonoscopy needs to be rescheduled or patients may be referred for alternative tests [20, 21].

CIR was reported as routinely recorded in only $62 \%$ of institutions. In addition, using the ESGE definition of CIR, which requires both written and photo-documentation, only $73.4 \%$ of procedures met requirements, which is short of the $\geq 90 \%$ minimum standard. However, when considering just written documentation, $94.7 \%$ reported reaching the cecum, almost reaching the $\geq 95 \%$ target standard.

Overall, PDR can be considered as a surrogate for ADR and is easier to monitor, because it is automatically collated by colonoscopists and institutions while generating procedure reports and/or billing codes, making it more practical to measure than ADR, even if less robust. Although our data show that PDR is more commonly recorded, it seems even this parameter falls short of the recommended standard, with only a minority of practitioners (47\%) and institutions (42\%) routinely recording the quality measure. When looking at the procedure forms, in terms of PDR, at least one polyp was detected in $40.5 \%$ of qualifying procedures, being borderline with the $\geq 40 \%$ ESGE minimum standard of screening and diagnostic colonoscopies performed in those aged 50 years or older. Initiatives such as education, adequate training, creating awareness, feedback, and colonoscopy quality benchmarking have been shown to contribute to improvement in these parameters [22-25].

Retraction time is recorded by only $60 \%$ of practitioners. Procedure data indicate the mean withdrawal time was $7.8 \pm$ 3.1 minutes, which reached the minimum standard (i.e. mean 6 minutes) defined by Kaminski et al [12]. Although we did not directly measure ADR, our data show that it was routinely recorded by only $34 \%$ of practitioners and in $29 \%$ of institutions. When considering the role of ADR as a universal key quality indicator, this is quite disappointing and might be one of the limiting factors for ADR underperformers.

Measurement of complication rate only partially entered routine practice: the collected responses showed that although during-procedure complications are usually recorded by the majority of institutions ( $83 \%$ ), almost one of two institutions (56\%) do not record post-procedure complications. This is comparable to the results by Adler et al [26]. This substantial under-recording probably reflects difficulty in monitoring patients after the procedure and the lack of availability of methods that allow the identification of a late complication.

Patient feedback, to enhance patient experience and colonoscopy quality, is important, and should be routinely monitored with adequate feedback mechanisms in place $[12,13]$. However, only a minority of institutions (25\%) record patient satisfaction and this merits further evaluation. Such underperformance in terms of recording of patient experience could be related to cultural issues (at least in some countries) as well as to logistic limitations related to the collection of patient feedback.

An important strength of this study is its size, both in terms of the number of colonoscopies analyzed and the Europe-wide coverage of the survey. Many colonoscopy quality studies are either single-center or restricted to a small number of colonoscopists. However, we accept that both the present study and the questionnaires have some limitations. The current findings are not based on consecutive reporting and a selection bias for those procedures recorded cannot be ruled out. Nevertheless, it reflects real-world data and can provide an efficient method to monitor colonoscopy quality measures both at an institutional and endoscopist level, with the aim to support initiatives and improve clinical practice standards. It identifies the quality measures that are adhered to, and how effective they can be in driving standards. Another important limitation of the present study is the self-selection of endoscopists across Europe for participation in the survey rather than random selection. It is debatable whether this self-selection bias might have selected a subgroup of endoscopists not representative of the general endoscopist population, leading to better results than in the general population of endoscopists. The same limitations, however, apply to all measures of voluntary quality control.

It is noteworthy that the publication of the ESGE PMs occurred after this version of the questionnaires was compiled; therefore, there are some areas in which the ECQI measures do not exactly match those specified by the ESGE [12].

In general, looking at the picture coming from the present study, we should admit that quality measures for colonoscopy are far from being routinely recorded in clinical practice. Performance measurement is the first step in a process aimed at improving quality in colonoscopy. Further steps include the identification of underperformers, of the barriers that need to be addressed, and subsequent reevaluation after corrective interventions have taken place. Measurement of performance parameters is the prerequisite without which concrete im- 
provement actions cannot be developed. Initiatives such as education, creating awareness, and training should be implemented to contribute to the overall improvement of colonoscopy. The final goal should be to improve quality, reducing gaps between clinical practice and evidence.

\section{Conclusions}

In conclusion, our findings clearly show areas in need of quality improvement and the importance of promoting quality monitoring throughout the colonoscopy procedure. They also underscore the necessity of regularly recording individual quality parameters to measure daily performance against well-established recommendations and evaluate their wider dissemination and adoption.

\section{Acknowledgements}

The ECQI Group is grateful for the continued financial support provided by Norgine Ltd. In October 2016, the ECQI Group became an independent working party, free to obtain funding from any reputable source. While it is not possible to acknowledge everyone individually, we are indebted and grateful to all of those who took the time to complete our questionnaires and provide an insight into their real-world practice. We would like to thank the practitioners who contributed to the ECQI dataset from the following institutions. In Belarus: the NN Alexandrov National Cancer Centre of Belarus; in Denmark: Herlev Hospital, Kirurgisk Klinik Frederikssund, Kirurgisk Klinik Syddanmark, Odense University Hospital Svendborg Sygehus; in Finland: Tampere University Hospital; in Germany: Allgemeines Krankenhaus Celle AKH, Gemeinschaftspraxis für Gastroenterologie und Innere Medizin Aschaffenburg, Internisten am Dominikanerplatz Würzburg, Klinikum Aschaffenburg-Alzenau, MathiasSpital Rheine, Sankt Elisabeth Hospital Gütersloh; in Hungary: Markusovszky University Teaching Hospital, University of Pécs, University of Szeged; in Italy: Centro di Riferimento Oncologico IRCCS, CTO and Sirai Hospitals Sardinia, Fondazione Poliambulanza Brescia, Fondazione Policlinico Gemelli Rome, Policlinico Umberto I "Sapienza" University of Rome; in Portugal: Centro Hospitalar do Baixo Vouga Aveiro, Centro Hospitalar TondelaViseu, Centro Hospitalar e Universitário de Coimbra, Endocentro - Idealmed UHC Coimbra, Portuguese Oncology Institute Coimbra, ULS Guarda; in Romania: Gastroenterology and Hepatology Center -TVM- Cluj-Napoca, Prof. Dr. Octavian Fodor Regional Institute of Gastroenterology and Hepatology, Research Center of Gastroenterology and Hepatology University of Medicine and Pharmacy of Craiova; in Russia: Yaroslavl Regional Cancer Hospital; in Spain: Hospital Álvaro Cunqueiro Vigo, Hospital Clínico Universitario Virgen de la Arrixaca, Hospital General Universitario de Alicante, Hospital del Mar Barcelona, Hospital Nisa Sevilla Aljarafe, Hospital Universitario de Fuenlabrada, Hospital Universitario Central de Asturias, Hospital Universitari i Politècnic La Fe Valencia; in Sweden: Ängelholm Hospital, Blekinge Hospital Karlshamn-Karlskorna, Capio St Göran's Hospital Stockholm, Central Hospital Karlstad, Centralsjukhuset Kristianstad, GHP Stockholm Gastro Center, Helsingborg Hospital,
Institute of Medicine Huddinge Karolinska Hospital Stockholm, Karolinska University Hospital Stockholm, Skåne University Hospital Malmö, South Älvsborg Hospital Borås, Specialistläkarna i Lund, Sunderby Hospital Luleå, University Hospital Linköping, Ystad Hospital; in the United Kingdom: Barnsley District General Hospital, Doncaster Royal Infirmary, Mid Yorkshire NHS Trust Grange Medical Centre, Royal Liverpool and Broadgreen University Hospitals, The Royal Infirmary of Edinburgh.

\section{Competing interests}

Dr. Spada is a consultant and advisory board participant for Norgine, AlfaSigma, Medtronic, Given Imaging, Covidien, Olympus, Intromedic, and AnX Robotica. A. Koulaouzidis reports material support from IntroMedic/SynMedUK, Jinshan/Aquilant; honoraria from Ferring UK, Dr Falk Pharma UK; travel support from Norgine, Jinshan/Aquilant; advisory board Dr Falk Pharma UK, Tillots; Given ${ }^{\circledR}$ Imaging Ltd/ESGE research grant 2011; cofounder AJM Med-i-caps Ltd. P. Amaro reports consultancy and advisory board participant for Norgine. L. Brink reports consultancy \& advisory board participant AMBU; travel support from Norgine. W. Fischbach reports consultancy and advisory board participant to Norgine; speaking - Abbott, Bio Merieux, Falk; advisory speaking - Aptalis, Fresenius Biotech, Pfizer; advisory - Boehringer Ingelheim, med update. M. Hünger reports travel support from Norgine. R. Jover reports consultancy - Norgine, MSD, GI Supply, CPP Pharma. U. Kinnunen reports travel support from Norgine. A. Ono reports travel support from Norgine. B. Amlani reports employee of Norgine. E. Toth reports consultancy and advisory board participant for Norgine. A. Agrawal, G. Antonelli, C. Hassan, Á. Patai, S. Pecere, L. Petruzziello, J.F. Riemann, L. Fuccio declare that they have no conflict of interest. Dr. Staines received fees for statistical services and Ann L. Stringer received fees for manuscript preparation from the ECQI Secretariat.

\section{Funding}

Funding from Norgine Ltd was received by Aspen Medical Media for provision of ECQI Secretarial support and by Pharmatelligence, for management of the questionnaire database. Award ID: NA

\section{References}

[1] Siegel R, Naishadham D, Jemal A. Cancer statistics, 2012. CA Cancer ] Clin 2012; 62: 10-29

[2] Edwards BK, Ward E, Kohler BA et al. Annual report to the nation on the status of cancer, 1975-2006, featuring colorectal cancer trends and impact of interventions (risk factors, screening, and treatment) to reduce future rates. Cancer 2010; 116: 544-573

[3] Kaminski MF, Regula J, Kraszewska E et al. Quality indicators for colonoscopy and the risk of interval cancer. N Engl J Med 2010; 362: 1795-1803

[4] Corley DA, Jensen CD, Marks AR et al. Adenoma detection rate and risk of colorectal cancer and death. N Engl J Med 2014; 370: 12981306

[5] Anderson JC, Butterly LF. Colonoscopy: quality indicators. Clin Transl Gastroenterol 2015; 6: e 77

[6] Allen JI. Quality measures for colonoscopy: where should we be in 2015? Curr Gastroenterol Rep 2015; 17: 10 
[7] Barclay RL, Vicari J], Doughty AS et al. Colonoscopic withdrawal times and adenoma detection during screening colonoscopy. N Engl J Med 2006; 355: 2533-2541

[8] Chen SC, Rex DK. Endoscopist can be more powerful than age and male gender in predicting adenoma detection at colonoscopy. Am J Gastroenterol 2007; 102: 856-861

[9] Yabroff KR, Borowski L, Lipscomb J. Economic studies in colorectal cancer: challenges in measuring and comparing costs. J Natl Cancer Inst Monogr 2013; 2013: 62-78

[10] Van Cutsem E, Borràs JM, Castells A et al. Improving outcomes in colorectal cancer: where do we go from here? Eur J Cancer 2013; 49: 2476-2485

[11] von Karsa L, Patnick J, Segnan N et al. European guidelines for quality assurance in colorectal cancer screening and diagnosis: overview and introduction to the full supplement publication. Endoscopy 2013; 45: 51-59

[12] Kaminski MF, Thomas-Gibson S, Bugajski M et al. Performance measures for lower gastrointestinal endoscopy: a European Society of Gastrointestinal Endoscopy (ESGE) Quality Improvement Initiative. Endoscopy 2017; 49: 378-397

[13] Valori R, Cortas G, de Lange T et al. Performance measures for endoscopy services: a European Society of Gastrointestinal Endoscopy (ESGE) Quality Improvement Initiative. Endoscopy 2018; 50 : 1186-1204

[14] Rembacken B, Hassan C, Riemann JF et al. Quality in screening colonoscopy: position statement of the European Society of Gastrointestinal Endoscopy (ESGE). Endoscopy 2012; 44: 957-968

[15] Riemann JF, Demedts I, Agrawal A et al. ECQI Group: Improving standards in colonoscopy through a practice level audit tool [PO160]. United European Gastroenterol ] 2015; 3: (Suppl. 05): A191

[16] Jover R, Agrawal A, Amaro P et al. Pilot results of the ECQI self-assessment questionnaire to evaluate quality in colonoscopy in Europe
[P0165]. United European Gastroenterol ] 2016; 05: (Suppl. 05): A213-A214

[17] Calderwood AH, Jacobson BC. Comprehensive validation of the Boston Bowel Preparation Scale. Gastrointest Endosc 2010; 72: 686-692

[18] Pontone S, Hassan C, Maselli R et al. Multiple, zonal and multi-zone adenoma detection rates according to quality of cleansing during colonoscopy. United European Gastroenterol J 2016; 4: 778-783

[19] Radaelli F, Paggi S, Hassan C et al. Split-dose preparation for colonoscopy increases adenoma detection rate: a randomised controlled trial in an organised screening programme. Gut 2017; 66: 270-277

[20] Wang L, Sprung BS, DeCross AJ et al. Split-dose bowel preparation reduces the need for early repeat colonoscopy without improving adenoma detection rate. Dig Dis Sci 2018; 63: 1320-1326

[21] Murphy D, Jenks M, McCool R et al. A systematic review and cost analysis of repeat colonoscopies due to inadequate bowel cleansing in five European countries. Expert Rev Pharmacoecon Outcomes Res 2019; 19: 701-709

[22] Kaminski MF, Anderson J, Valori R et al. Leadership training to improve adenoma detection rate in screening colonoscopy: a randomised trial. Gut 2016; 65: 616-624

[23] Coe SG, Crook JE, Diehl NN et al. An endoscopic quality improvement program improves detection of colorectal adenomas. Am J Gastroenterol 2013; 108: 219-227

[24] Corley DA, Jensen CD, Marks AR. Can we improve adenoma detection rates? A systematic review of intervention studies. Gastrointest Endosc 2011; 74: 656-665

[25] Brenner H, Altenhofen L, Kretschmann J et al. Trends in adenoma detection rates during the first 10 years of the German screening colonoscopy program. Gastroenterology 2015; 149: 356-366

[26] Adler A, Lieberman D, Aminalai A et al. Data quality of the German screening colonoscopy registry. Endoscopy 2013; 45: 813-818 